\title{
A Measurement Method for Dispersion in Optical Fiber Communication with Long Distance
}

\author{
Xue-zhao Zheng \\ College of Energy, Xi'an University of Science and Technology, Xi'an 710054, China \\ 1libero2014@163.com
}

\begin{abstract}
With the development of the telecommunication, optical fiber has been widely used. It has the characteristics of long distance transmission and large capacity, but it also has dispersion exists. The dispersion can be divided into some types. Mode dispersion plays a major role in multimode fiber, while chromatic dispersion or intramodal dispersion are the main mechanism in single mode fiber. Generally, it is quite important to test the dispersion and know the dispersion characteristics. The theoretical basis and experimental device, which are both based on the interference principle, have been introduced in detail. The device is used to measure the fiber dispersion characteristics of optical fiber and the results shows the validity of the theory and the designed test device.
\end{abstract}

Keywords: optical fiber, dispersion, modified method, measurement

\section{Introduction}

Different light components (different models or different frequency) usually travel at different speeds in optical fiber, and this phenomenon is known as dispersion [1-3]. Dispersion is an important optical characteristics in the optical fiber. Dispersion [4-6] will broaden optical pulse, and cause serious limit on the transmission capacity and optical fiber bandwidth. For multimode fiber [7-10], mode dispersion plays a major role, this means different modes travel at different velocities to lead to the dispersion. For the single mode fiber [11-15], chromatic dispersion or intramodal dispersion is the main mechanism, and this is to say that the dispersion is caused by the different frequencies on different transmission speed. Due to the limitation of the mode dispersion, the transmission rate cannot exceed $100 \mathrm{Mb} / \mathrm{s}$. Generally speaking, single-mode fiber is more superior to multimode fiber. In the practical application, single-mode fiber is widely used and it is more important to consider the dispersion of single mode fiber.

The main dispersion in single mode is the material dispersion [16-18] and waveguide dispersion [19-22]. Material dispersion is that variation of frequency transmission will lead to the variation of refractive index and cause the variation of transmission constant and group velocity.

Waveguide dispersion is due to the variation of frequency resulted in changes of the waveguide parameters and cause the variation of transmission constant and group velocity. Intramode dispersion is mainly due to the polychromatic source. In addition, two orthogonal linear polarization modes exist in single-mode fiber transmission. Due to the non-circular symmetry, optical fiber, edge stress, and fiber bending and so on, polarization mode dispersion will appear in the transmission.

Dispersion is an important feature in optical structure fiber. Various methods [23-25] have been developed for numerical calculation in dispersion characteristics for microstructure, but 
the experimental methods in measurement of micro structural fiber dispersion are still inadequate. The results of theoretical calculations should be validated by experimental test. For the reason of not perfect in the production of optical fiber, the actual properties should still be studied. So in order to express the performance of the optical performance, characterization of optical fiber is urgently needed.

In this paper, from the view of summarizing the existing method and put forward new methods to measure the fiber dispersion. The main contribution is the establishment of a measurement method for dispersion, and the remainder of the paper is shown as the following: optical fiber and its model is introduce in section 2. Some measurement methods are summarized in section 3. New method is proposed in section 4 . The experiment is shown in section 5 and the conclusion is described in section 6 .

\section{Dispersion and Compensation Methods}

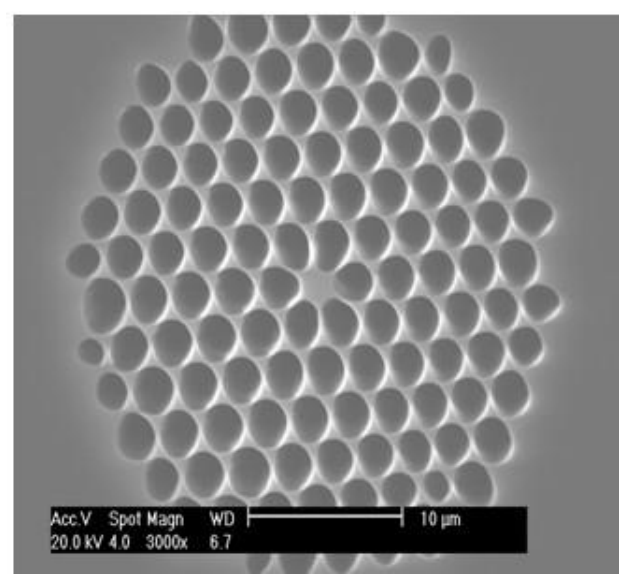

Figure 1. Scanning Electron Micrographs of PCF

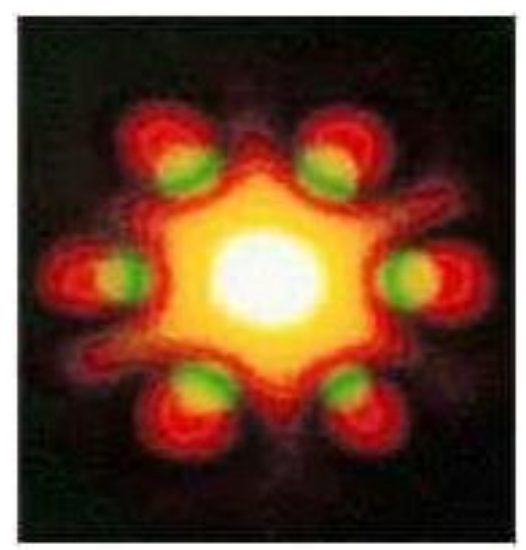

Figure 2. Transmission Effect of PCF

The basic equations of pulse transmission in single mode fiber can be described as the following:

$$
\frac{\partial A}{\partial z}+\frac{i}{2} \beta_{2} \frac{\partial^{2} A}{\partial T^{2}}-\frac{1}{6} \beta_{3} \frac{\partial^{3} A}{\partial T^{3}}=0
$$


Where, $A$ is the slowly varying amplitude; $z$ is the transmission distance; $T$ is time; $\beta_{2}$ is the group velocity dispersion (GVD) or second order dispersion coefficient and it is the main factors of pulse broadening; $\beta_{3}$ is the coefficient of high order dispersion (also called third order dispersion). Compared with the second order dispersion, effect of third order dispersion on the pulse is usually small. When $\left|\beta_{2}\right|>1 \mathrm{ps}^{2} / \mathrm{km}, \beta_{3}$ can be negligible. The solution of the function can be obtained as:

$$
A(z, T)=\frac{1}{2 \pi} \int_{-\infty}^{+\infty} A(0, \omega) \exp \left[\frac{i}{2} \beta_{2} z \omega^{2}-i \omega T\right] d \omega
$$

Where, $A(0, \omega)$ is the Fourier transform of $A(0, T)$. Then, we can know that the optical signal distortion caused by dispersion is determined by phase coefficient $\exp \left(\frac{i \beta_{2} \omega^{2}}{2}\right)$.

Dispersion of single-mode optical fiber per unit length can be obtained by the following formula:

$$
\delta=-\frac{\lambda}{c} \frac{d^{2} n(\lambda)}{d \lambda^{2}}-\frac{n \Delta}{c} V \frac{d^{2} V b}{d \lambda^{2}}
$$

Where, $c$ is the light velocity; $V$ is the normalized frequency of optical fiber transmission; $b$ is the normalized propagation constant. First item at the right of the formula (3) depends on the refractive index of material, which is known as the material dispersion; second item is relative to the fiber waveguide properties, which is called waveguide dispersion. Chromatic dispersion coefficient of common single mode optical fiber in $1550 \mathrm{~nm}$ window is about 16 $\mathrm{ps} /(\mathrm{nm} \cdot \mathrm{km})$ and its dispersion can reach $1600 \mathrm{ps} / \mathrm{nm}$. For a system with capacity of $10 \mathrm{Gbit} / \mathrm{s}$, the tolerance of maximum dispersion is $1000 \mathrm{ps} / \mathrm{nm}$. Therefore, in order to make the system run normally, it must be compensated.

\section{Measurement Methods}

Photonic Crystal Fibers (PCF), which is also called Micro-Structured Fibers (MSF), are widely paid attention in recent years. Complex refraction rate distribution exists on the cross section of PCF. In the PCF, stomata with different arrangements are included, and its size is in roughly the same order of magnitude with the wavelength of light wave and throughout the entire length of optical devices. The light wave will be limited in the center region of the fiber with a low refractive index.

The concept of photogenic crystals first appeared in 1987. Some researchers proposed that the electronic band gap of semiconductor has similar periodic dielectric structure with optic, and one of the most promising fields is the application of photonic crystal in optical fiber. It is mainly relating to the periodic high refractive index optical fiber with microstructure, which is normally consists of stomata with the silicon dioxide as the background material. This new type of optical waveguide has two types: (1) fiber with high index refractive core layer and surrounded structure by two-dimensional photonic crystal cladding. These fibers have similar properties with conventional optical fiber, and its principle is that the waveguide is formed by total internal reflection (TIR). Compared to conventional refraction index guiding, it is allowed for photonic crystal cladding with higher refractive index. Therefore, it is important to note that internal total reflection photonic crystal fiber (TIR-PCFs) is actually not depend on the Photonic Band Gap (PBG) effect; (2) fiber with Photonic Band Gap (PBG) effect. The fiber controls the beam in the core layer with this effect. The optical fiber (PBG-PCFs) exhibits considerable performance, and the most important is controlling and guiding the 
beam transfer in the core layer with a lower refractive index than that of cladding refractive index.

Dispersion measurement of fiber dispersion is measuring the time delay of different frequencies light pulse travel the same distance, or measuring the pulse phase of different frequencies with corresponding frequency domain. There are many methods in measuring fiber dispersion, which mainly include pulse delay method, phase shift method and interferometric method.

\subsection{Pulse Delay Method}

Time delay method is one of the simplest methods. In this method, dispersion value will be acquired according to the polynomial fitting by measuring the time delay of different frequencies light pulse.

$$
D=\frac{1}{L} \frac{d \tau_{g}}{d \lambda}=-\frac{d}{d \lambda}\left(\frac{1}{V_{g}}\right)=-\frac{2 \pi c}{\lambda^{2}} \frac{d^{2} \beta}{d \omega^{2}}=-\frac{2 \pi c}{\lambda^{2}} \beta_{2}
$$

Where $D$ is the dispersion coefficient, and it means the time delay of light signal with unit wavelength interval transmitting unit distance difference. Its unit is $\mathrm{ps} / \mathrm{nm} \cdot \mathrm{km}$. Factor $\beta$ is group velocity dispersion parameter:

$$
\beta^{2}=\frac{d^{2} \beta}{d \omega^{2}}
$$

And it determines pulse broadening when it is in the propagation of the optical fiber.

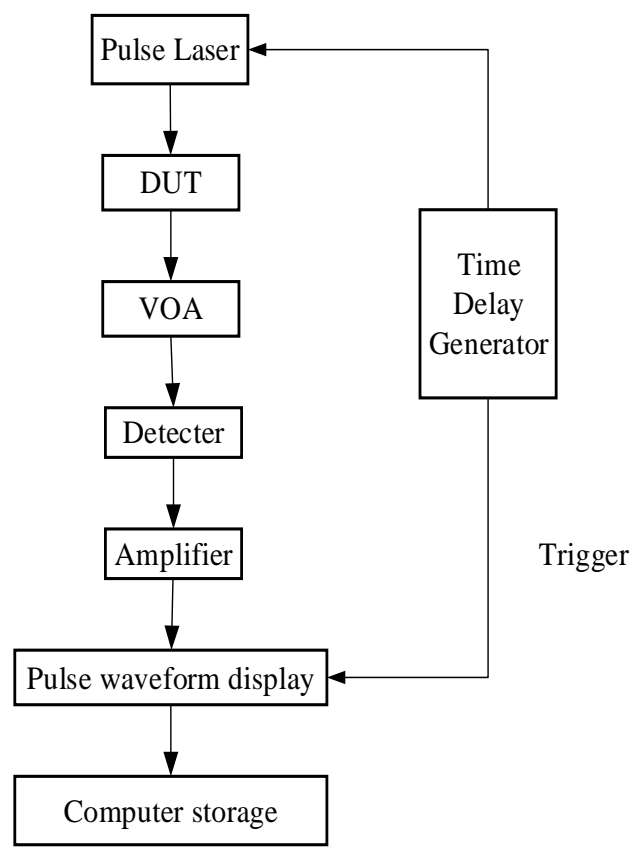

Figure 3. Pulse Delay Method

Figure 3 shows a typical time delay measurement device. Where, DUT is fiber (equipment) under test. VOA is the adjustable optical attenuator. The light source is laser diode or fiber Raman laser with a series of different wavelengths. Fiber Raman laser stimulates simultaneously several different wavelengths of light pulse according to Raman scattering effect. The first pulse signal delay will be set as a reference, and time delay of rest optical 
pulse signals will be compared with this reference value. Time delay difference and corresponding wavelength would be fitted and dispersion parameters would be acquired.

In practical applications, time delayed method, which is based on optical time domain reflectometer (OTDR), is used to measure the delay dispersion. Its time resolution is 50ps. It has the advantages of simplicity method, low cost device, and long measurable fiber distance, while the disadvantages are inaccuracy time delay and corresponding wavelength. In addition, due to limitation of light source, number of sampling points is not enough and it also affects the precision of measurement.

\subsection{Phase Shift Method}

The most frequently used method of measuring optical fiber dispersion is the phase shift method and differential phase shift method. Phase shift method is according to compare the measured values and phase reference value to obtain the phase difference and then acquire the dispersion value. According to the different reference types of phases, it can be classified into electric signals and optical signals reference.

In electric signals reference phase shift methods, one is the modulation phase shift technique, as shown in Figure 4.

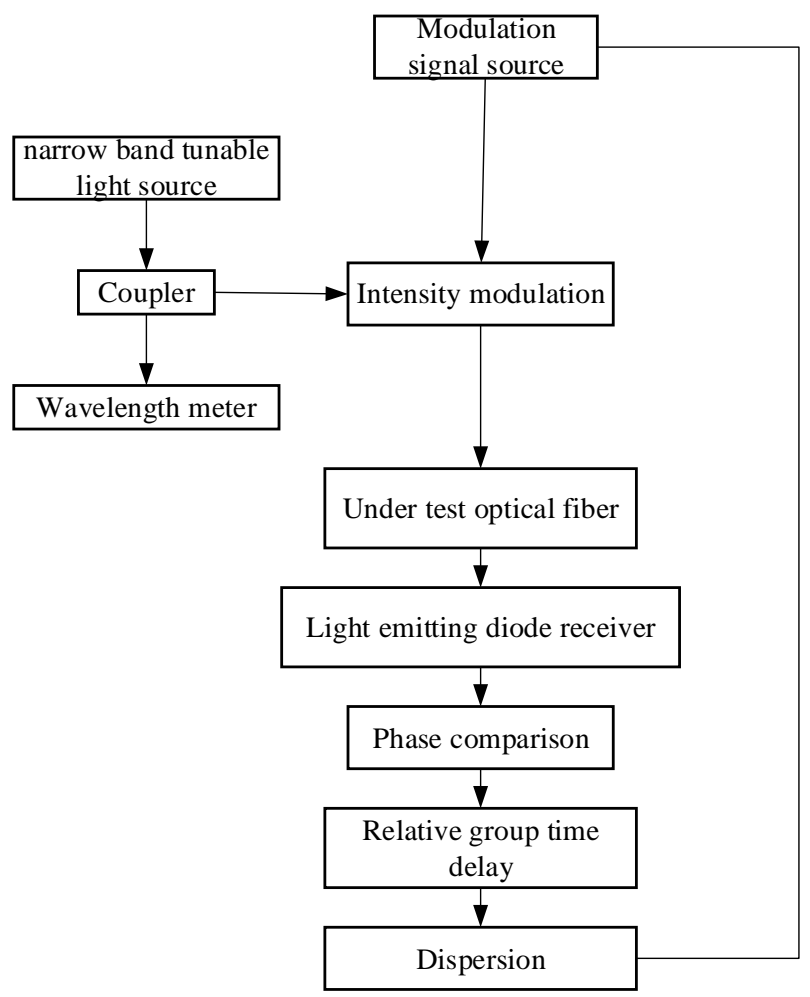

Figure 4. Phase Shift Method

Signal generator will carry out the modulation for the intensity of light output from the narrow band tunable light source according to the external modulator.

The modulated light signal with information will cross through the optical fiber, and the transmission signal will be detected by photoelectric. Then vector voltmeter will be used to measure the modulation phase difference between the received signal and the modulation 
signal source. This measurement method can be measured for any wavelength, and group delay differential between adjacent intervals will be:

$$
\Delta \tau \lambda=\frac{\phi_{\lambda+\Delta \lambda / 2}-\phi_{\lambda-\Delta \lambda / 2}}{2 \pi f_{m}}
$$

Where, $\lambda$ is the central wavelength in wavelength interval, $f_{m}$ is the modulation frequency and the unit is MHz. Based on the measured data, curve graph of group time delay with variation of wavelength can be obtained. After the linear fitting, relationship between $\Delta \tau \lambda$ and $\lambda$ can be obtained. Then get derivation of $\lambda$, and pulse broadening caused by dispersion will be acquired.

\subsection{Interferometric Method}

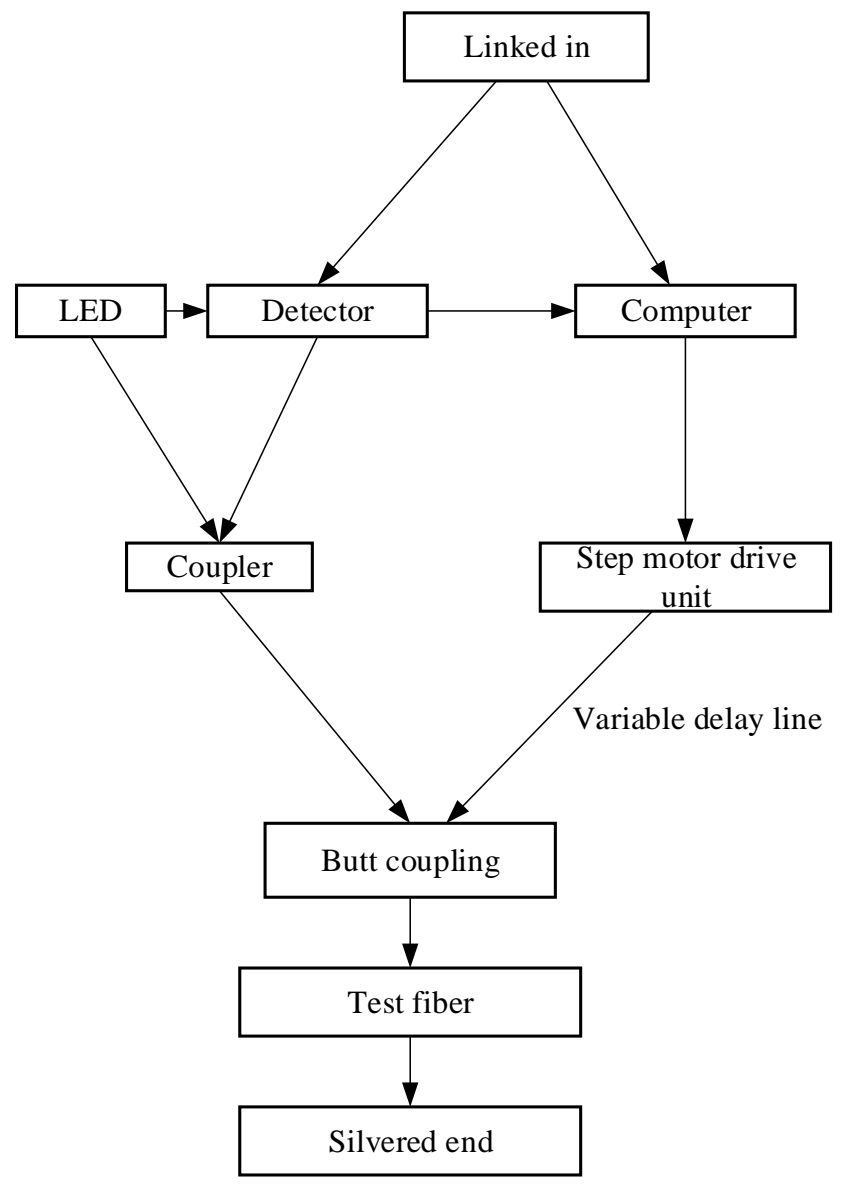

Figure 5. Interferometric Method

Interferometic method is a measurement of dispersion without expensive instruments. Use the Mach-Zehnder Interferometer or its arm as the under tested optical fiber arm and reference arm. According to changing the reference arm length, intensity information of interference fringes of light from the two arms will be obtained:

$$
I(d) \propto F T^{-1}\left[R(\omega) e^{-i \beta(\omega) L}\right](d / c)
$$

Where, $d$ is the length variation of reference arm; $L$ is the length of optical fiber under tested; $R(\omega)$ is the parameter relevant to the transfer function of arms; $\beta(\omega)$ is the transfer constant for different frequency. From the formula listed before, the phase information 
- $\beta(\omega) L$ can be obtained when the intensity information of interference fringes is processed with Fourier transformation. Due to the limitation of Fourier transformation, we can't get the actual value and just get the relative size of the phase in the frequency domain.

A modified Michelson interference based fiber dispersion measurement device is shown in Figure 5. When the length of the reference fiber arm is changed, the refractive index is also changed. Then, time delay can be obtained as follows:

$$
\begin{gathered}
\tau(\omega)=\frac{N_{0}(\omega) z_{0}}{c}+\Gamma \frac{\delta z}{c} \\
\Gamma=N_{0}\left\{1-N_{0}^{2}\left[P_{12}-v\left(P_{11}+P_{22}\right)\right] / 2\right\}
\end{gathered}
$$

The $\delta_{z}$ is the length variation of optical fiber, $P_{i j}$ is the elasto optical coefficient of fiber material, $v$ is the Poisson ratio of the fiber material.

\section{Modified Measurement Methods}

Based on existing interferometry method, a modified method has been proposed to measure the dispersion of optical fiber. Test device measurement based on the modified method is shown in Figure 6.

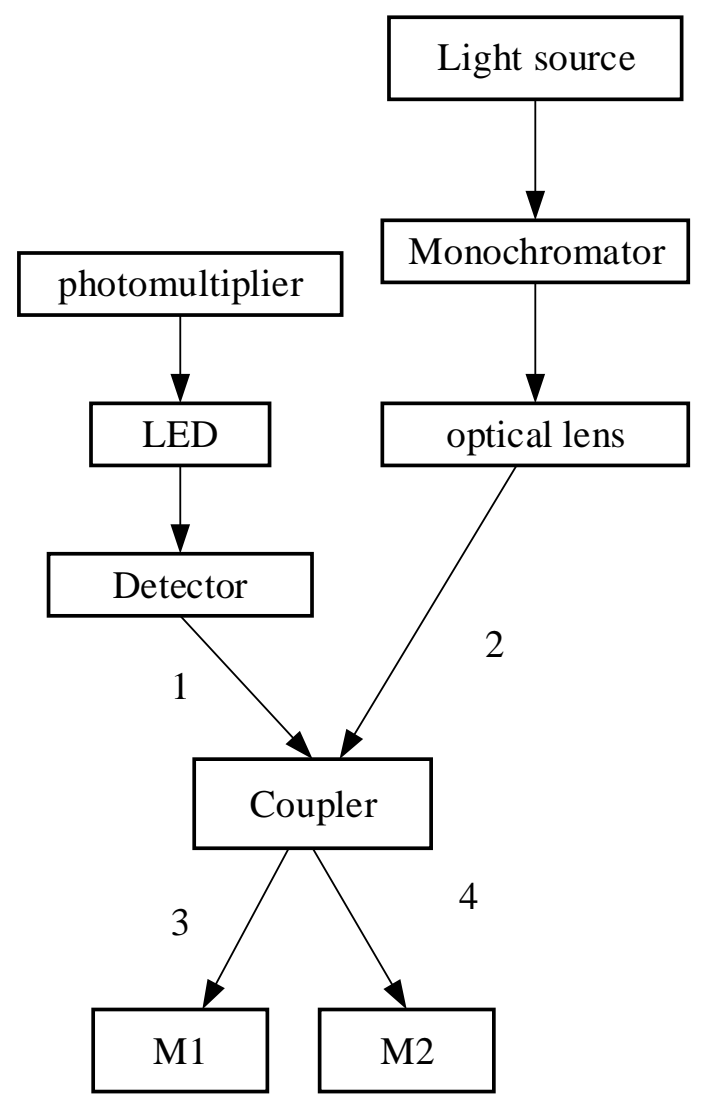

Figure 6. Comparison of the Algorithms

Structure diagram of single core with total internal reflection used in the test is with parameters listed as follows: the optical fiber cladding air hole diameter $d$ is $3.05 \mu \mathrm{m}$; air hole pitch $\Lambda=4.2 \mu \mathrm{m}$, and the packet is composed of 8 layers of air hole. 
The reference arm is set with air, and the measuring arm is the micro structural fiber. When the receiving end of photomultiplier tube receives the interference spectrum, repeated measurements for the interference graph will be adopted and then the data collected will be averaged. The curve will be smoothed in order to denoising.

The coupler has four arms. The third arms are the reference arm, and the fourth arm is measuring arm of optical fiber. M1 and M2 are the reflector. The light source in the experiment is LTH/75W bromine tungsten lamp, and its spectral range of $250 \sim 2500 \mathrm{~nm}$. The photomultiplier tube (PMT) is the PMTH- S1 side window photomultiplier, and spectral response range is $300 \sim 950 \mathrm{~nm}$. Grating monochromator is SBP500 with the minimum resolution of $0.1 \mathrm{~nm}$.

The first and second arm in the testing system is solid quartz arms, while the third and fourth arms are hollow pipes and connected with the coupler. In the two arms, reference optical fiber and under test optical fiber are respectively arranged. The middle part of the coupler is composed of about four pieces of quartz arm. The light source of bromine tungsten lamp will emit white light and then the light passes through a monochromator to output light with single wavelength and passing through the lens then coupled to the quartz arm 1 . This light passes through the $2 * 2$ couplers and then goes into the arm 3 and 4 with the under measured optical fiber and reference optical fiber. Then, light reflected by M1, M2 will meet the coherence conditions.

Spectral intensity received at photomultiplier tube of interference arm photoelectric 2, which is injected from interferometer arm 1, can be written as:

$$
\begin{gathered}
I(\omega)=\left|E_{f}(\omega)+E_{s}(\omega)\right|^{2} \approx 1+\cos \Phi(\omega) \\
\Phi(\omega)=\beta_{f}(\omega) L-\beta_{s}(\omega) d
\end{gathered}
$$

Where, $E_{f}, E_{s}, \beta_{f}, \beta_{s}$ is the dielectric field, which is the propagation constant along the fiber arm and free space. $L$ and $D$ are the optical fiber arm length and free space length. If we give the series form of $\beta_{f}$, then $\phi(\omega)$ can be described as follows:

$$
\begin{aligned}
\phi= & \phi_{0}+\left[\beta_{f}\left(\omega_{0}\right) L-d / c\right]\left(2 \pi c / \lambda-2 \pi c / \lambda_{0}\right)+ \\
& \frac{1}{2} \beta_{2}\left(\omega_{0}\right) L\left(2 \pi c / \lambda-2 \pi c / \lambda_{0}\right)^{2}+ \\
& \frac{1}{6} \beta_{3}\left(\omega_{0}\right) L\left(2 \pi c / \lambda-2 \pi c / \lambda_{0}\right)^{3}+
\end{aligned}
$$

Where, $\phi_{0}$ is constant, $C$ is the speed of light in vacuum, $\beta_{2}$ is the total group velocity dispersion, $\beta_{3}$ is the three order dispersion of optical fiber. Therefore, the appropriate selection of the optical wavelength intensity, $\beta_{2}$ and $\beta_{3}$ can be obtained.

\section{Test Results}

Figure 7 to Figure 9 shows the experimental test values of dispersion parameter. Figure 10 shows the difference between Experimental value $D$ and theoretical calculation value of dispersion parameter. The difference shows the error, and the main reason may be the fiber structure is not perfect. The error includes structural asymmetry and heterogeneity lead to the calculation error and test accuracy. However, measured and calculated results are very similar. This means the validity of the proposed test device. 


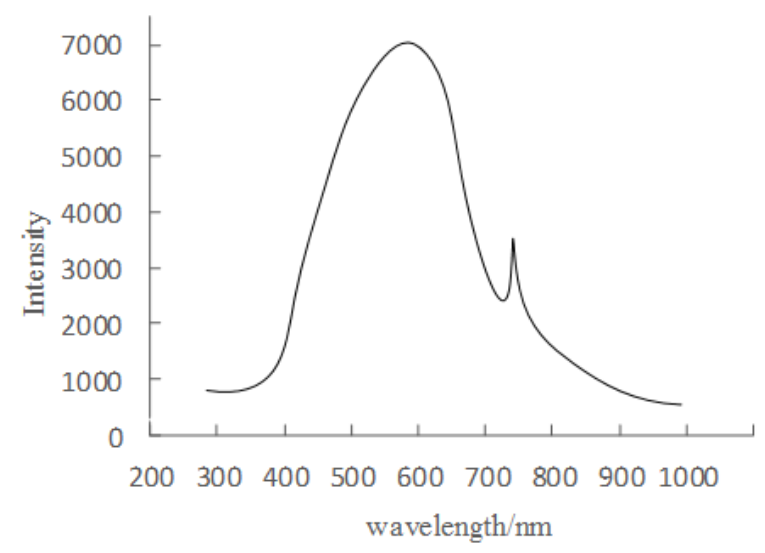

Figure 7. Interferogram of Single Core Fiber and Micro Structure of the Air

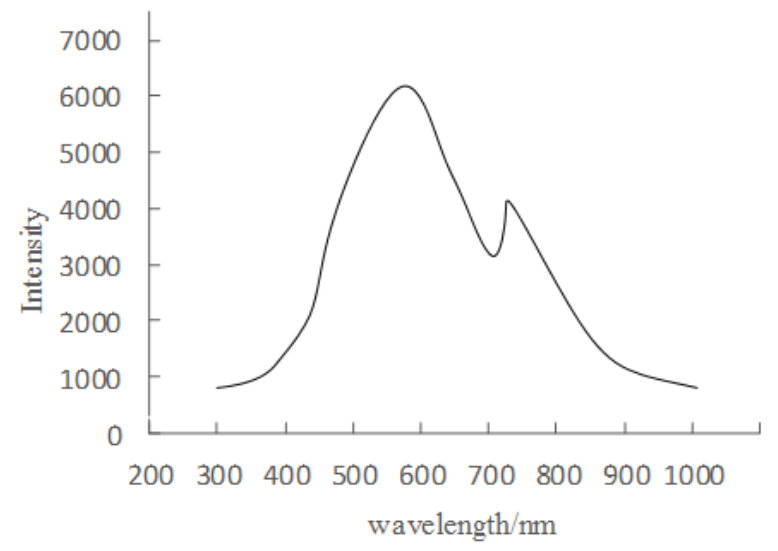

Figure 8. Interferogram of Crystal and Air

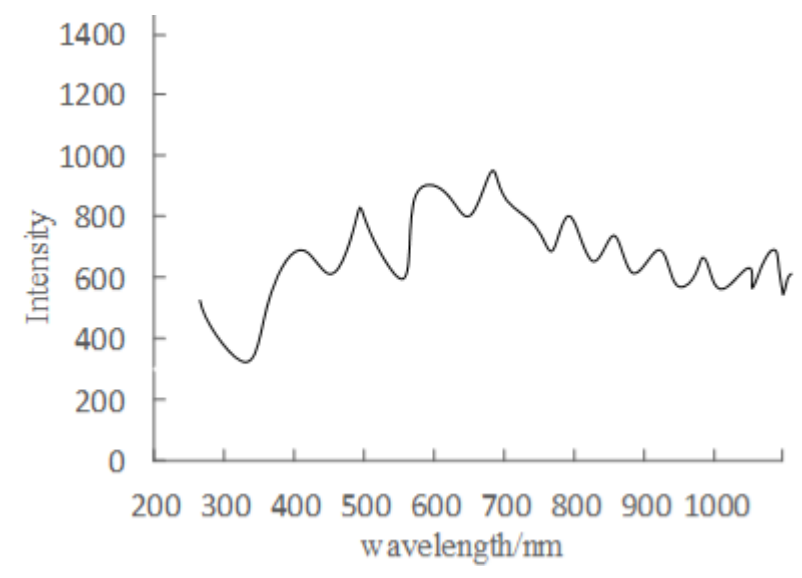

Figure 9. Coherent Curve of Figure 7 and 8 


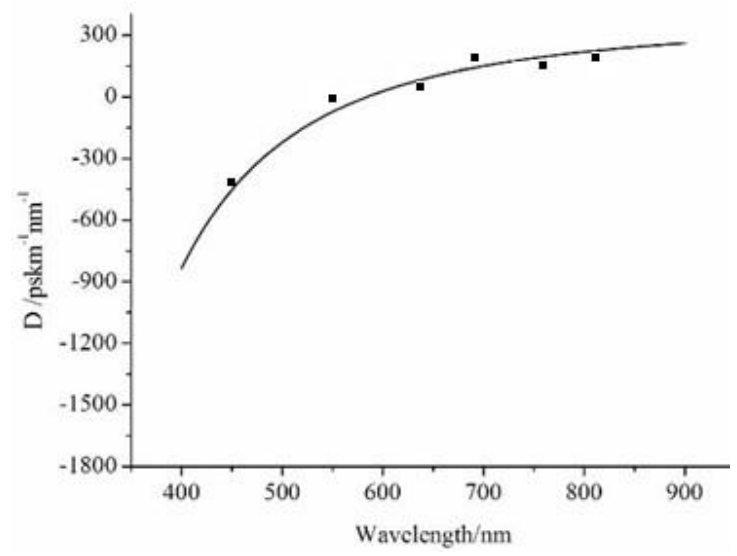

Figure 10. Comparison of Theoretical and Experimental Results

\section{Conclusions}

Dispersion exists in optical fiber in the telecommunication. It is an important optical characteristics in the optical fiber and will broaden optical pulse. Mode dispersion plays a major role in multimode fiber, while chromatic dispersion or intramodal dispersion is the main mechanism in single mode fiber. Now, single mode fiber is widely used, so it is quite important to test the dispersion and know the dispersion characteristics.

This paper introduces a test method and designs a test device. The theoretical basis and experimental device have been introduced in detail. The device is used to measure the fiber dispersion characteristics of optical fiber and the results shows the validity of the theory and the designed test device.

\section{Acknowledgments}

Natural Science Foundation of China (51134019); Shaanxi Provincial Department of Education Science Research Program (2013JK0862)

\section{References}

[1] Y.-Q. Huang, J.-E. Song, J.-X. Li, Y.-H. Xia, X. Chen and X.-M. Ren, "Dispersion simulation of long optical fiber by using fiber Bragg gratings: Theory and Experiment”, Journal of Optoelectronics Laser, vol. 14, no. 3, (2003), pp. 266-270.

[2] D. H. Spadoti, B.-H. V. Borges and M. A. Romero, "Novel microstructured optical fiber design for broadband dispersion compensation", SBMO/IEEE MTT-S International Microwave and Optoelectronics Conference Proceedings, (2007), pp. 702-706.

[3] Y. Li, T. Xu, D. Jia, W. Jing, H. Hu, J. Yu and Y. Zhang, "Dynamic dispersion compensation in a $40 \mathrm{~Gb} / \mathrm{s}$ single-channeled optical fiber communication system”, Acta Optica Sinica, vol. 27, no. 7, (2007), pp. 11611165.

[4] S. Wabnitz and C. Finot, "Theory of parabolic pulse propagation in nonlinear dispersion-decreasing optical fiber amplifiers", Journal of the Optical Society of America B: Optical Physics, vol. 25, no. 4, (2008), pp. 614-621.

[5] W. Cao, Y. Wang and S. Liu, "Dispersion and nonlinearity compensation in optical fiber communication systems by optical phase conjugation incorporated pulse prechirp", Acta Optica Sinica, vol. 32, no. 9, (2012).

[6] C. Finot, S. Wabnitz, A. Guryanov and A. Sysoliatin, "Parabolic pulse generation with dispersion decreasing optical fiber", Optics InfoBase Conference Papers, Nonlinear Photonics, (2007).

[7] F. Poletti and P. Horak, "Description of ultrashort pulse propagation in multimode optical fibers", Journal of the Optical Society of America B: Optical Physics, vol. 25, no. 10, (2008), pp. 1645-1654.

[8] B. Barviau, S. Randoux and P. Suret, "Spectral broadening of a multimode continuous-wave optical field propagating in the normal dispersion regime of a fiber", Optics Letters, vol. 31, no. 11, (2006), pp. 1696-1698. 
[9] E. D. Diebold, N. K. Hon, Z. Tan, J. Chou, T. Sienicki, C. Wang and B. Jalali, "Giant tunable optical dispersion using chromomodal excitation of a multimode waveguide", Optics Express, vol. 19, no. 24, (2011), pp. 23809-23817.

[10] H. Itoh, T. Urakami, S.-I. Aoshima and Y. Tsuchiya, "Femtosecond pulse delivery through long multimode fiber using adaptive pulse synthesis", Japanese Journal of Applied Physics, Part 1: Regular Papers and Short Notes and Review Papers, vol. 45, no. 7, (2006), pp. 5761-5763.

[11] T. K. Gangopadhyay, A. Halder, S. Das, M. C. Paul, M. Pal, M. Salza and G. Gagliardi, "Fabrication of tapered single mode fiber by chemical etching and used as chemical sensor based on evanescent field absorption", Proceedings of SPIE - The International Society for Optical Engineering, vol. 8173, (2011).

[12] D. F. Murphy, N. Healy, K. T. O'Mahoney and C. D. Hussey, "Adiabatically expanded single-mode optical fibers for reduced optical power density and enhanced reliability", 2012 Optical Fiber Communication Conference and Exposition and the National Fiber Optic Engineers Conference, OFC/NFOEC 2012, (2012).

[13] T. Nappez, E. Ghibaudo, P. Rondeau, J.-P. Schlotterbeck, J.-E. Broquin, "Broad-area laser diode with stable single-mode output and wavelength stabilization", Proceedings of SPIE - The International Society for Optical Engineering, vol. 8264, (2012).

[14] A. B. Socorro, I. D. Villar, J. M. Corres, F. J. Arregui and I. R. Matias, "Tapered single-mode optical fiber pH sensor based on lossy mode resonances generated by a polymeric thin-film”, IEEE Sensors Journal, vol. 12, no. 8, (2012), pp. 2598-2603.

[15] W. Luo, C. Geng, Y.-Y. Wu, Y. Tan, Q. Luo, H.-M. Liu, X.-Y. Li, "Experimental demonstration of singlemode fiber coupling using adaptive fiber coupler", Chinese Physics B, vol. 23, no. 1, (2014).

[16] A. Rostami and S. Makouei, "Modified W-type single-mode optical fiber design with ultra-low, flattened chromatic dispersion and ultra-high effective area for high bit rate long haul communications", Progress In Electromagnetics Research C, vol. 12, (2010), pp. 79-92.

[17] R. Freund, D.-D. Gro, M. Seimetz, L. Molle and C. Caspar, "30 Gbit/s RZ-8-PSK transmission over 2800 km standard single mode fibre without inline dispersion compensation”, OFC/NFOEC 2008 - 2008 Conference on Optical Fiber Communication/National Fiber Optic Engineers Conference, (2008).

[18] Y. H. Wang, "All-wave non-zero dispersion-flattened single mode fibers", Asia Optical Fiber Communication and Optoelectronic Exposition and Conference, AOE 2006, (2006).

[19] K. Gao and Z. Fang, "A new method of measuring the waveguide dispersion factor and the thermo-optic coefficient of long-period fiber gratings", Optics Communications, vol. 244, nos. 1-6, (2005), pp. 227-231.

[20] N.-K. Chen and S. Chi, "Spectral characteristics of side-polished endlessly single-mode photonic crystal fiber: Waveguide dispersion", 2006 Optical Fiber Communication Conference, and the 2006 National Fiber Optic Engineers Conference, 2006, (2006).

[21] I. V. Neves, A. L. Topa and M. G. Neves, "Waveguide dispersion in a strongly biaxial anisotropic fiber: An analytical solution", Journal of Lightwave Technology, vol. 30, no. 14, (2012), pp. 2262-2270.

[22] J. A. Mores Jr., G. N. Malheiros-Silveira, H. L. Fragnito and H. E. Hernández-Figueroa, "Efficient calculation of higher-order optical waveguide dispersion", Optics Express, vol. 18, no. 19, (2010), pp. 1952219531.

[23] K. Rafa, D. MacIej and P. Krassimir, "Waveguiding losses of micro-structured fibres-plane wave method revisited", Optical and Quantum Electronics, vol. 39, nos. 4-6, (2007), pp. 469-479.

[24] N. Farahat, R. Mittra and J. Carrion, "Analysis of optical fiber waveguides using the body of revolution version of the finite difference time domain method", Microwave and Optical Technology Letters, vol. 50, no. 1, (2008), pp. 213-216.

[25] D. Lateva, G. Toshev and S. Terzieva, "New equivalent-step-index method for determination of Gaussian deviation of single-mode fibers", Proceedings of the IEEE International Conference on Electronics, Circuits, and Systems, vol. 2, (1996), pp. 920-922.

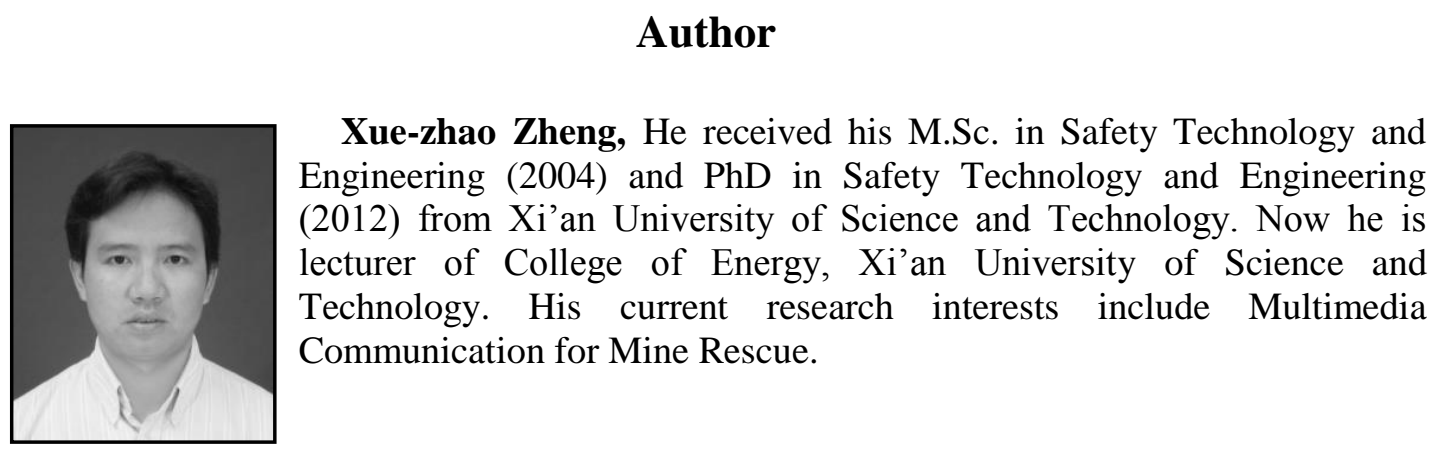


International Journal of Future Generation Communication and Networking Vol. 7, No. 6 (2014) 